\title{
Applications of rigged Hilbert spaces in quantum mechanics and signal processing
}

\author{
E. Celeghini, ${ }^{1,2, a)}$ M. Gadella, ${ }^{2, b)}$ and M. A. del Olmo ${ }^{2, c)}$ \\ ${ }^{1}$ Dipartimento di Fisica, Università di Firenze and INFN-Sezione di Firenze, \\ 150019 Sesto Fiorentino, Firenze, Italy \\ ${ }^{2}$ Departamento de Física Teórica, Atómica y Óptica and IMUVA, Universidad de Valladolid, \\ Paseo Belén 7, 47011 Valladolid, Spain
}

(Received 9 March 2016; accepted 28 June 2016; published online 25 July 2016)

\begin{abstract}
Simultaneous use of discrete and continuous bases in quantum systems is not possible in the context of Hilbert spaces, but only in the more general structure of rigged Hilbert spaces (RHS). In addition, the relevant operators in RHS (but not in Hilbert space) are a realization of elements of a Lie enveloping algebra and support representations of semigroups. We explicitly construct here basis dependent RHS of the line and half-line and relate them to the universal enveloping algebras of the Weyl-Heisenberg algebra and $s u(1,1)$, respectively. The complete sub-structure of both RHS and of the operators acting on them is obtained from their algebraic structures or from the related fractional Fourier transforms. This allows us to describe both quantum and signal processing states and their dynamics. Two relevant improvements are introduced: (i) new kinds of filters related to restrictions to subspaces and/or the elimination of high frequency fluctuations and (ii) an operatorial structure that, starting from fix objects, describes their time evolution. Published by AIP Publishing. [http://dx.doi.org/10.1063/1.4958725]
\end{abstract}

\section{INTRODUCTION}

It is commonly accepted that the starting point of quantum mechanics on the real line $\mathbb{R}$ is the Weyl-Heisenberg algebra, spanned by set of observables $\{X, P, \mathbb{I}\}$. Here $X$ and $P$ represent the position and momentum observables, respectively, and $\mathbb{I}$ the identity. In the usual representation of this algebra as operators on the Hilbert space of functions $L^{2}(\mathbb{R}), X$ is the multiplication operator and $P$ is the derivative multiplied by $-i$. The second standard ingredient of the real line quantum space is the Fourier transform that connects the coordinate representation, where the wave functions depend on the position, to the momentum representation, where the wave functions depend on the momentum. ${ }^{1,2}$

In the present paper, we use an approach based on Hermite functions (HF) introduced as transition devices connecting the two continuous bases of eigenvectors of $X,\{|x\rangle, x \in \mathbb{R}\}$, and $P,\{|p\rangle, p \in \mathbb{R}\}$, with a discrete orthonormal basis $\{|n\rangle, n \in \mathbb{N}\}$ (where $\mathbb{N}$ is the set of non-negative integers, $0,1,2, \ldots$ ) in one-to-one relation to the Hermite functions. ${ }^{3-5}$

Thus one adds a new operator, the so called number operator $N$ which reads the degree of Hermite functions, to the previously mentioned Weyl-Heisenberg algebra. This provides a realization of the projective algebra $i o(2)$ where $N$ defines a discrete basis in $L^{2}(\mathbb{R})$ and the space of operators on $L^{2}(\mathbb{R})$ is isomorphic to the universal enveloping algebra (UEA) of $i o(2)$, here denoted as UEA $[i o(2)]$. Elements of the orthonormal basis $\{|n\rangle, n \in \mathbb{N}\}$ are eigenvectors of the number operator $N$.

As continuous basis make sense on rigged Hilbert spaces (RHS) only, this tool emerges naturally here. On the other hand, $\{|n\rangle, n \in \mathbb{N}\}$ spans the Hilbert space which plays a central role in the RHS. Therefore, RHS is a correct device to use continuous and discrete bases simultaneously.

\footnotetext{
a) celeghini@fi.infn.it

b) manuelgadella1@gmail.com

c) olmo@fta.uva.es
} 
In addition, one of the points of interest of the present formulation comes up its connection with the fractional Fourier transform, which has been extensively used in signal processing (see Refs. 6 and 7 and references therein). Moreover, formal properties of the scalar product space $L^{2}(\mathbb{R})$ and of the set of operators, $O(\mathbb{R})$, acting on it can be extended to all signal processing applications.

Since this is a central tool in our analysis, let us define in brief the concept of RHS. ${ }^{8-14}$ A rigged Hilbert space or Gelfand triple is a set of three vector spaces,

$$
\Phi \subset \mathcal{H} \subset \Phi^{\times},
$$

where (i) $\mathcal{H}$ is an infinite dimensional separable (i.e., with a countable orthonormal basis) Hilbert space; (ii) $\Phi$ is a topological vector space endowed with a topology finer than the Hilbert space topology, this implies in particular that the canonical injection $\Phi \longmapsto \mathcal{H}$ is continuous. In addition, $\Phi$ is dense on $\mathcal{H}$ with the Hilbert space topology. ${ }^{15}$ Finally, (iii) $\Phi^{\times}$is the dual space of $\Phi$, i.e., the space of linear (or antilinear) continuous mappings from $\Phi$ into the set of complex numbers $\mathbb{C}$, and it is endowed with a topology compatible with the dual pair $\left(\Phi, \Phi^{\times}\right) .{ }^{16}$

Rigged Hilbert spaces have been used in order to implement the Dirac formulation of quantum mechanics. ${ }^{9-14,17,18}$ Continuous bases are well defined in the dual $\Phi^{\times}$. The action of a functional $F \in \Phi^{\times}$on a vector $\varphi \in \Phi$ is usually written as $\langle\varphi \mid F\rangle$ in order to keep up with the Dirac notation. ${ }^{19}$ Since we assume that the scalar product on Hilbert space is antilinear to the left, we shall assume the antilinearity of the elements in $\Phi^{\times}$.

The mathematical properties of RHS have been studied with detail in the previous references. Once they have been established, we realize that the structure of RHS is quite simple (as we do not need to insist in the foundations of these properties, only on their existence) and useful. In particular, it allows us for manipulations in both discrete and continuous bases, even with continuous bases on different representations. ${ }^{20}$

Once we have established the definition of RHS, let us proceed with the objective of our analysis. First of all, let us recall the well known fact that the projective algebra $i o(2)$, spanned by the generators $\langle X, P, N, \mathbb{I}\rangle$ as above, is isomorphic to the algebra of the harmonic oscillator $\left\langle H, a, a^{\dagger}, \mathbb{I}\right\rangle$, which is often represented as contained in the UEA of the Weyl-Heisenberg algebra. ${ }^{21,22}$

In the sequel we see that the infinite dimensional $U E A[i o(2)]$ is a very rich object and a set of relevant structures will emerge on $L^{2}(\mathbb{R})$ and on operators on $L^{2}(\mathbb{R})$. In particular, as the $U E A[i o(2)]$ contains infinitely many copies of $i o(2)$, we find infinitely many operators that close $i o(2)$. Furthermore, all these structures admit an abstract setting for which the previous one is just one explicit representation.

One interesting property shows that the subspace structure on $L^{2}(\mathbb{R})$ derived from the algebra may also be obtained from the fractional Fourier transform. ${ }^{6}$ As a matter of fact, the eigenvectors of the fractional Fourier transform are the Hermite functions, exactly as for the ordinary Fourier transform. For each natural number $k>0$, a fractional Fourier transform can be constructed with the property that its eigenvalues are the roots of unity of order $k$, each root defining one subspace of $L^{2}(\mathbb{R})$.

As discussed in Sec. III, this construction is not exclusive for $\mathbb{R}$. In fact the whole discussion can be repeated for $\mathbb{R}^{+} \equiv[0, \infty)$. Moreover, starting with the Hermite functions and the relations between the Hermite functions and the generalized Laguerre functions, we can construct two Fourier-like transforms $\mathcal{T}^{ \pm}$with appropriate eigenvectors defined as functions on the half-line.

It is also possible to extend this formalism to $\mathbb{R}^{n}$ and also to spherical coordinates. This will be discussed in a forthcoming paper.

The Hermite and Laguerre functions are bases of spaces of real and complex functions defined on $\mathbb{R}$ and $\mathbb{R}^{+}$, respectively, that allow a formal description of both real functions of signal processing and wave (complex) functions of quantum physical systems.

New kinds of filters, based on either restrictions to subspaces of $L^{2}(\mathbb{R})\left(L^{2}\left(\mathbb{R}^{+}\right)\right)$or to low values of the parameter $n$ or both together, can be introduced to clean the signal (or the wave) functions from noise or unwanted effects.

Moreover the operatorial space introduced on $\mathbb{R}\left(\mathbb{R}^{+}\right)$and related to UEA $[i o(2)](\operatorname{UEA}[\operatorname{su}(1,1)])$ allows us to relate different functions and, in particular, to describe the formal time-evolution of a state to another one in terms of filters or interaction introduced. 
This paper is organized as follows. In Section II, we discuss the action on the quantum line $\mathbb{R}$ in different subsections. In Subsection II A we use Hermite functions to construct the rigged Hilbert space with both discrete and continuous bases. We analyze the relations of position and momentum operators with the ladder operators of the suitable discrete basis in Subsection II B. In Subsection II $\mathrm{C}$, we show that the space of relevant operators acting on the quantum $\mathbb{R}, O(\mathbb{R})$, is contained in $U E A[i o(2)]$. We present a complete analysis of the substructures on $\mathbb{R}$ and $O(\mathbb{R})$ in Subsection II D. Finally, in Subsection II E we use the fractional Fourier transform to exhibit again, from a different point of view, how the vector space of actions on $\mathbb{R}$ is articulated into subspaces. In Section III, the precedent discussion is repeated for the actions on the positive semiaxis $\mathbb{R}^{+}$. However, in this second approach there are a couple of fundamental differences with respect to the former: (i) The use of Laguerre functions instead of Hermite functions, which is discussed in III A. As shown in III B, the crucial Lie algebra is not longer io [2] but instead $s u(1,1)$. (ii) The form of the Fourier-like transforms and their respective fractional extensions, described in III C, are those corresponding specifically to the action on the half-line $\mathbb{R}^{+}$. They play a similar role as the Fourier transforms on $\mathbb{R}$ obtained in Section II. Some conclusions close the manuscript.

\section{WAVE FUNCTIONS AND SIGNALS ON $\mathbb{R}$}

\section{A. Rigged Hilbert space on $\mathbb{R}$ and Hermite functions}

To begin with, let us describe the effect of the unitary irreducible representations of the one dimensional translation group $T^{1}$ on a continuous basis. Let us recall that $T^{1} \simeq \mathbb{R}$ has only one infinitesimal generator $P$ and that the action of $P$ and the action of the group on the continuous basis $\{|p\rangle, p \in \mathbb{R}\}$ are, respectively, given by

$$
P|p\rangle=p|p\rangle, \quad R(x)|p\rangle=e^{-i p x}|p\rangle,
$$

for all $p \in \mathbb{R}$ and for all $x \in T^{1}$. This continuous basis has the following properties:

$$
\left\langle p \mid p^{\prime}\right\rangle=\sqrt{2 \pi} \delta\left(p-p^{\prime}\right), \quad \frac{1}{\sqrt{2 \pi}} \int_{-\infty}^{\infty}|p\rangle\langle p| d p=\mathbb{I} .
$$

The conjugate basis $\{|x\rangle, x \in \mathbb{R}\}$ is the basis of eigenvectors of the operator $X$. It can be obtained as the Fourier transform of $\{|p\rangle\}$

$$
\begin{aligned}
& |x\rangle=\frac{1}{\sqrt{2 \pi}} \int_{-\infty}^{\infty} e^{-i p x}|p\rangle d p, \\
& \left\langle x \mid x^{\prime}\right\rangle=\sqrt{2 \pi} \delta\left(x-x^{\prime}\right), \\
& \frac{1}{\sqrt{2 \pi}} \int_{-\infty}^{\infty}|x\rangle\langle x| d x=\mathbb{I} .
\end{aligned}
$$

As is well known, the operators $X, P$, and $\mathbb{I}$ close the Weyl-Heisenberg algebra. Now, we move to a non-standard approach related to the ray representations ${ }^{21-23}$ of the inhomogeneous orthogonal algebra $i o(2)$. In this representation, along $X$ and $P$, we have an operator $N$ related to the index $n \in \mathbb{N}$ of the Hermite functions

$$
\psi_{n}(x):=\frac{e^{-x^{2} / 2}}{\sqrt{2^{n} n ! \sqrt{\pi}}} H_{n}(x),
$$

where $H_{n}(x)$ are the Hermite polynomials. The Hermite functions have well known properties coming from the fact that they form an orthonormal basis in $L^{2}(\mathbb{R})$

$$
\begin{aligned}
& \int_{-\infty}^{\infty} \psi_{n}(x) \psi_{m}(x) d x=\delta_{n m}, \\
& \sum_{n=0}^{\infty} \psi_{n}(x) \psi_{n}\left(x^{\prime}\right)=\delta\left(x-x^{\prime}\right) .
\end{aligned}
$$


The operators $X$ and $P$ in one side and $N$ on the other have a quite different property: while $X$ and $P$ have a non-countable continuous spectrum covering the whole real line, $N$ has a countable spectrum. This corresponds to the fact that any representation of $X$ and $P$ has continuous bases as above, while a representation of $N$ has a countable basis. The latter can be easily constructed from the continuous basis as

$$
|n\rangle:=(2 \pi)^{-1 / 4} \int_{-\infty}^{\infty} \psi_{n}(x)|x\rangle d x,
$$

for $n=0,1,2, \ldots$ That the vectors $\{|n\rangle, n \in \mathbb{N}\}$ complete a countable orthonormal basis as one may straightforwardly check from the definition (4)

$$
\langle n \mid m\rangle=\delta_{n m}, \quad \sum_{n=0}^{\infty}|n\rangle\langle n|=\mathbb{I} .
$$

Thus, we have three bases in our representation, for which the relations among them are easily established. The crucial point to prove this relation is the fact that the Hermite functions (2) are eigenfunctions of the Fourier transform in the following sense:

$$
\begin{aligned}
{\left[\mathcal{F} \psi_{n}\right](p) } & \equiv \widetilde{\psi}_{n}(p) \\
& =\frac{1}{\sqrt{2 \pi}} \int_{-\infty}^{\infty} e^{i p x} \psi_{n}(x) d x \\
& =i^{n} \psi_{n}(p) .
\end{aligned}
$$

Then, we can complete the relations between discrete and/or continuous bases adding to the first relation in (1) and to the definition (4) the following ones:

$$
\begin{aligned}
& |p\rangle=\frac{1}{\sqrt{2 \pi}} \int_{-\infty}^{\infty} e^{i p x}|x\rangle d x, \\
& |n\rangle=i^{n}(2 \pi)^{-1 / 4} \int_{-\infty}^{\infty} \psi_{n}(p)|p\rangle d p, \\
& |x\rangle=(2 \pi)^{1 / 4} \sum_{n=0}^{\infty} \psi_{n}(x)|n\rangle, \\
& |p\rangle=(2 \pi)^{1 / 4} \sum_{n=0}^{\infty} i^{n} \psi_{n}(p)|n\rangle,
\end{aligned}
$$

where expression (7) just comes from (1); (8) from (1), (4), and (6); (9) from (3) and (4); etc., For any ket $|f\rangle$ which can be formally written in the form

$$
|f\rangle=\frac{1}{\sqrt{2 \pi}} \int_{-\infty}^{\infty} d x f(x)|x\rangle,
$$

with

$$
f(x):=\langle x \mid f\rangle=\sum_{n=0}^{\infty} a_{n} \psi_{n}(x),
$$

we can, with the help of all of the above, establish the following relations:

$$
\begin{aligned}
|f\rangle & =\frac{1}{\sqrt{2 \pi}} \int_{-\infty}^{\infty} d p \tilde{f}(p)^{*}|p\rangle \\
& =(2 \pi)^{-1 / 4} \sum_{n=0}^{\infty} a_{n}|n\rangle,
\end{aligned}
$$


where

$$
\tilde{f}(p)^{*}=\langle p \mid f\rangle=\sum_{n=0}^{\infty}(-i)^{n} a_{n} \psi_{n}(p)
$$

and

$$
\begin{aligned}
a_{n} & =(2 \pi)^{1 / 4}\langle n \mid f\rangle=\int_{-\infty}^{\infty} d x \psi_{n}(x) f(x) \\
& =i^{n}(2 \pi)^{-1 / 4} \int_{-\infty}^{\infty} d p \psi_{n}(p) \tilde{f}(p)^{*} .
\end{aligned}
$$

Thus, the wave functions $f(x), \tilde{f}(p)$ and the sequence of complex numbers $\left\{f_{n}\right\}$ describe the quantum state $|f\rangle$ as coefficients relative to three distinct bases, $\{|x\rangle\},\{|p\rangle\}$, and $\{|n\rangle\}$. Contrary to the latter, which is infinite countable, the two former bases are non-countable.

Meanwhile countable bases, also called orthonormal bases or even complete orthonormal systems, like $\{|n\rangle\}$ are well defined in Hilbert spaces, in order to have continuous bases, we need a superstructure such as a rigged Hilbert space. Furthermore, the rigged Hilbert space structure can give the necessary tools to pass from one representation to another. ${ }^{18}$

\section{B. Position and momentum operators in $R H S[\mathbb{R}]$}

This situation is quite suitable to support a representation of the algebra of the harmonic oscillator for which the generators are the number operator $N$ (or $H=N+1 / 2$ ), the creation $a^{\dagger}$ and annihilation $a$ operators and the identity I. All these operators act naturally on the basis $\{|n\rangle\}$ as follows:

$$
\begin{array}{rlrl}
a^{\dagger}|n\rangle & =\sqrt{n+1}|n+1\rangle, & & \mathbb{I}|n\rangle=|n\rangle, \\
a|n\rangle=\sqrt{n}|n-1\rangle, & & N|n\rangle=n|n\rangle .
\end{array}
$$

On the other hand, being true that

$$
X=\frac{1}{\sqrt{2}}\left(a+a^{\dagger}\right), \quad P=\frac{-i}{\sqrt{2}}\left(a-a^{\dagger}\right),
$$

these operators act naturally on this basis, but they cannot be diagonalized on a Hilbert space, as continuous bases make no sense on a Hilbert space.

If a Hilbert space does not serve to describe quantum mechanics encompassing all the features described above, there exists another space that can do it. As we shall see, it is a rigged Hilbert space that makes the job.

The point of departure is the Schwartz space, $\mathcal{S}$, of all complex functions on the real line that are derivable at all orders and at all points and such that they, their product for any power of the variable and their derivatives go to zero at the infinity faster than the inverse of any polynomial. Functions belonging to the Schwartz space are square integrable and $\mathcal{S}$ is a vector space dense in $L^{2}(\mathbb{R})$ with the norm topology. In addition, $\mathcal{S}$ has a natural complete metric topology (Fréchet), which is finer than the topology inherited from $L^{2}(\mathbb{R})$. The dual of $\mathcal{S}$ is represented by $\mathcal{S}^{\times}$, which is the space of the tempered distributions. ${ }^{8,24}$

For any function $f(x) \in L^{2}(\mathbb{R})$, we can define one and only one tempered distribution associated to it, which we shall denote equally by $f(x)$. Its action on any $g(x) \in \mathcal{S}$ is given by

$$
\langle f(x) \mid g(x)\rangle:=\int_{-\infty}^{\infty} f^{*}(x) g(x) d x .
$$

Thus, we can identify $f(x) \in L^{2}(\mathbb{R})$ with one and only one distribution. This identification gives sense to the second inclusion below

$$
\mathcal{S} \subset L^{2}(\mathbb{R}) \subset \mathcal{S}^{\times}
$$

This is the rigged Hilbert space that we use for the description of the harmonic oscillator. In the standard representation of the operators $X$ and $P$ as the multiplication operator $X f(x)=x f(x)$ and the derivation $P f(x)=-i d f(x) / d x$, this RHS has important advantages as follows: 
1. For any $f(x) \in \mathcal{S}$, both $X f(x)$ and $P f(x)$ are in $\mathcal{S}$. We say that $\mathcal{S}$ reduces both $X$ and $P$.

2. Both operators $X$ and $P$ are continuous on $\mathcal{S}$, when we endow $\mathcal{S}$ with its own Fréchet topology.

3. The operators $X$ and $P$ can be extended as linear operators on the dual $\mathcal{S}^{\times}$by means of these duality formulas:

$$
\begin{aligned}
& \langle X F \mid f(x)\rangle:=\langle F \mid X f(x)\rangle, \\
& \langle P F \mid f(x)\rangle:=\langle F \mid P f(x)\rangle,
\end{aligned}
$$

for all $F \in \mathcal{S}^{\times}$and for all $f(x) \in \mathcal{S}$.

For simplicity, we have also denoted by $X$ and $P$ their extensions as operators on $\mathcal{S}^{\times}$. These extensions are also linear. Furthermore, they are continuous under any topology on $\mathcal{S}^{\times}$compatible with the dual pair structure $\left\{\mathcal{S}, \mathcal{S}^{\times}\right\}$, in particular weak and strong topologies on $\mathcal{S}^{\times}$, which coincide in this case.

These duality formulas can be used to extend any operator $A$ for which the adjoint $A^{\dagger}$ is reduced by $\mathcal{S}$. In this case, the duality formula reads $\langle A F \mid f(x)\rangle=\left\langle F \mid A^{\dagger} f(x)\right\rangle, \forall f(x) \in \mathcal{S}$ and $F \in \mathcal{S}^{\times}$. Here, we have also denoted by $A$ its the extension to $\mathcal{S}^{\times}$. This property is general and can be used in any rigged Hilbert space.

Since $a$ and $a^{\dagger}$ are linear combinations of $X$ and $P$, they share with $X$ and $P$ the above properties, including their extensions to $\mathcal{S}^{\times}$via the above duality formula and all the operators (16) are well defined as continuous operators either on $\mathcal{S}$ or on $\mathcal{S}^{\times}$.

The most natural choice for the basis $\{|n\rangle\}$ is the sequence of normalized HF $\psi_{n}(x)$, which all belong to $\mathcal{S}$.

Then, how continuous bases appear in the present context? The answer is as follows: as a consequence of a theorem by Gelfand and Maurin, which serves for a rigorous justification of the Dirac formulation of quantum mechanics. Although not in its full generality or with its full consequences, this theorem could be given as in here: $8,14,18,25$

Theorem (Gelfand-Maurin).- Let $A$ be a self-adjoint operator, with domain $\mathcal{D}_{A}$, on a separable infinite dimensional Hilbert space $\mathcal{H}$. Then, there exists a subspace $\Phi \subset \mathcal{H}$ dense on $\mathcal{H}$ having the following properties:

1. The space $\Phi$ is a subspace of the domain $\mathcal{D}_{A}$ (otherwise, we could not apply the operator $A$ to the vectors in $\Phi$ ), so that $\Phi \subset \mathcal{D}_{A}$. In addition, $\Phi$ reduces $A$, a property that we may denote by writing $A \Phi \subset \Phi$.

2. We can endow $\Phi$ with a topology finer than the Hilbert space norm topology, such that $A$ be a continuous operator on $\Phi$. If we denote by $\Phi^{\times}$the dual of $\Phi$, we conclude that $\Phi \subset \mathcal{H} \subset \Phi^{\times}$is a rigged Hilbert space. This topology on $\Phi$ can be chosen to be nuclear. ${ }^{26}$ Infinite dimensional nuclear spaces share many of the properties of finite dimensional normed spaces. In particular the unit ball is compact. If $\Phi$ is nuclear the canonical injection $i: \Phi \mapsto \mathcal{H}$ admits a spectral decomposition in terms of series. This condition of nuclearity can be weakened. ${ }^{14}$

3. As a consequence of (1) and (2), $A$ can be extended to $\Phi^{\times}$. In addition, there exists a measure $d \mu(\lambda)$ defined on the Hilbert space spectrum of $A, \sigma(A)$, such that for almost all $\lambda \in \sigma(A)$ with respect to $d \mu(\lambda)$, there exists a functional $|\lambda\rangle \in \Phi^{\times}$such that $A|\lambda\rangle=\lambda|\lambda\rangle$.

4. For any pair of vectors $\psi, \varphi \in \Phi$ and any $n=0,1,2, \ldots$, we have the following spectral decomposition:

$$
\left\langle\psi \mid A^{n} \varphi\right\rangle=\int_{\sigma(A)} \lambda^{n}\langle\psi \mid \lambda\rangle\langle\lambda \mid \varphi\rangle d \mu(\lambda),
$$

where $\langle\lambda \mid \varphi\rangle=\langle\varphi \mid \lambda\rangle^{*}$ and the star means complex conjugation. Formula (19) is often written as

$$
A^{n}=\int_{\sigma(A)} \lambda^{n}|\lambda\rangle\langle\lambda| d \mu(\lambda)
$$

for $n=0,1,2, \ldots$ 
5. For any vector $\varphi \in \Phi$, we have

$$
\varphi=\int_{\sigma(A)}\langle\lambda \mid \varphi\rangle|\lambda\rangle d \mu(\lambda)
$$

As a matter of fact, (21) is nothing else than (19), where we have omitted $\psi$ and chosen $n=0$. This is the representation of $\varphi$ in the continuous basis $\{|\lambda\rangle\}$. In addition, the function $\varphi(\lambda):=\langle\lambda \mid \varphi\rangle$ is square integrable and it is customary to write (21) as

$$
\varphi=\int_{\sigma(A)} \varphi(\lambda)|\lambda\rangle d \mu(\lambda)
$$

In the situation under our consideration, $\Phi \equiv \mathcal{S}$ and $\mathcal{H} \equiv L^{2}(\mathbb{R})$, so that the rigged Hilbert space is given as $\mathcal{S} \subset L^{2}(\mathbb{R}) \subset \mathcal{S}^{\times}$. For both operators $X$ and $P$ the measure $d \mu(\lambda)$ is absolutely continuous with respect to the Lebesgue measure, so that it can be replace by this one and write $d x$ and $d p$, respectively. ${ }^{27}$ This means, in particular that for any functions $f(x)$ and $\tilde{f}(p)$ in $\mathcal{S}$, Fourier transform of each other, (22) gives (11) and the first formula in (13).

We are now in the position of justifying formally all relations (7)-(15). In fact,

(i) Let us consider the operator $X$. Since in this case $d \mu(\lambda)$ is absolutely continuous with respect to the Lebesgue measure on the line, now (20) has the following form (for obvious reasons we write $x$ instead of $\lambda$ ):

$$
X^{n}=\int_{-\infty}^{\infty} x^{n}|x\rangle\langle x| d x
$$

with $n=0,1,2, \ldots$ and $X|x\rangle=x|x\rangle$, for all real $x$. For $n=0$ we have an identity $\mathbb{I}$, which is the canonical identity from $\mathcal{S}$ into $\mathcal{S}^{\times}$. In general, this identity cannot be extended as an operator on $\mathcal{S}^{\times}$. However, if for some $|F\rangle \in \mathcal{S}^{\times}$we had a meaning ${ }^{28}$ for $\langle x \mid F\rangle$, then, II could be applied to $|F\rangle$.

In fact, $P$ admits an spectral decomposition similar to (23) of the form

$$
P^{n}=\int_{-\infty}^{\infty} p^{n}|p\rangle\langle p| d p,
$$

with $n=0,1,2, \ldots$ and $P|p\rangle=p|p\rangle$, for all real $p$. Furthermore, $\langle p \mid x\rangle$ is well defined as $e^{-i p x}$ for fixed $x$ and $p$ and also $\langle x \mid p\rangle=e^{i p x}$. Thus,

$$
\begin{aligned}
|p\rangle & =\mathbb{I}|p\rangle=\int_{-\infty}^{\infty}|x\rangle\langle x \mid p\rangle d x \\
& =\int_{-\infty}^{\infty} e^{-i p x}|x\rangle d x .
\end{aligned}
$$

(ii) If the kets $|n\rangle$ are represented by the normalized Hermite functions, $\psi_{n}(x)$, (8) is just (22), where we have taken $P$ for the operator $A$ and $\widetilde{\psi}_{n}(p)$ is the Fourier transform of $\psi_{n}(x)$. Note that also (4) is (22), where $X$ plays the role of $A$. Moreover, after (21), $\langle x \mid n\rangle=\psi_{n}(x)$ and $\langle p \mid n\rangle=\widetilde{\psi}_{n}(p)$ for $x$ and $p$ fixed, respectively.

(iii) As a consequence of the above comment, the formal identity ${ }^{29} \mathbb{I}=\sum_{n=0}^{\infty}|n\rangle\langle n|$ on $\mathcal{S}$ can be formally applied to some elements in $\mathcal{S}^{\times}$like $|x\rangle$ and $|p\rangle$. This gives (9) and (10). Note that Hermite functions are real. Being true that $\mathcal{S} \subset \mathcal{S}^{\times}$, all $|n\rangle \in \mathcal{S}^{\times}$.

(iv) Equation (11) and the first identity in (13), are nothing else than (22). The second identity in (13) is the span of $|f\rangle$ in terms of an orthonormal basis in $L^{2}(\mathbb{R})$.

(v) The first identity in (12) is indeed a definition and reflects the relation between (21) and (22). One has this and the last identity in (13), the second in (12) becomes trivial. Same comment about (14). Finally (15) is the scalar product on $L^{2}(\mathbb{R})$ and the Parseval identity.

(vi) So far, we have operated as no difference would exists between $|f\rangle, f(x) \in \mathcal{S}$, and $\langle x \mid f\rangle$. There is indeed a difference and to explain it, let us consider an abstract rigged Hilbert space $\Phi \subset \mathcal{H} \subset \Phi^{\times}$with the following properties: 
(a) There exists a unitary operator $U: \mathcal{H} \longmapsto L^{2}(\mathbb{R})$ with the property that the mapping: $U: \Phi \longmapsto \mathcal{S}$ is onto (it is one-to-one because it us unitary).

(b) The operator $U$ is a continuous mapping between $\Phi$ and $\mathcal{S}$, with their own topology, and its inverse $U^{-1}$ is also continuous.

As a consequence, $U$ can be extended as a continuous one-to-one mapping from the dual $\Phi^{\times}$onto the dual $\mathcal{S}^{\times}$with continuous inverse. This can be done through the following version of the duality formula:

$$
\langle U \varphi \mid U F\rangle=\langle\varphi \mid F\rangle, \quad \forall \varphi \in \Phi, \quad \forall F \in \Phi^{\times} .
$$

In this case, we say that the triplet $\mathcal{S} \subset L^{2}(\mathbb{R}) \subset \mathcal{S}^{\times}$is a representation of $\Phi \subset \mathcal{H} \subset \Phi^{\times}$, i.e.,

$$
\begin{array}{llllll}
\mathcal{S} & \subset & L^{2}(\mathbb{R}) & \subset & \mathcal{S}^{\times} \\
\uparrow U & & \uparrow U & & \uparrow U . \\
\Phi & \subset & \mathcal{H} & \subset & \Phi^{\times}
\end{array}
$$

On the other hand, $U^{-1} X U$ and $U^{-1} P U$ have the same properties on the triplet $\Phi \subset \mathcal{H} \subset \Phi^{\times}$as have $X$ and $P$ on $S \subset L^{2}(\mathcal{R}) \subset \mathcal{S}^{\times}$, respectively. It would be convenient to use the notation $|x\rangle$ and $|p\rangle$ for the eigenkets of $U^{-1} X U$ and $U^{-1} P U$, so that they are in $\Phi^{\times}$.

For each $x \in \mathbb{R}$, the action of $|x\rangle \in \Phi^{\times}$on $|f\rangle \in \Phi,\langle f \mid x\rangle$ is a complex number. When $x$ runs out the whole real line $\mathbb{R}$, the function $f(x):=\langle x \mid f\rangle=\langle f \mid x\rangle^{*}$ is a representation of $|f\rangle$.

Take $|x\rangle \in \Phi^{\times}$such that $U^{-1} X U|x\rangle \equiv X|x\rangle=x|x\rangle$. Obviously $|\tilde{x}\rangle:=U|x\rangle \in \mathcal{S}^{\times}$. Then,

$$
\begin{aligned}
X|\tilde{x}\rangle & =X U|x\rangle=U\left[U^{-1} X U\right]|x\rangle \\
& =U X|x\rangle=U(x|x\rangle)=x U|x\rangle=x|\tilde{x}\rangle .
\end{aligned}
$$

Also for any $|f\rangle \in \Phi, U|f\rangle$ is in the Schwartz space $\mathcal{S}$. Thus, the function $\langle\tilde{x}|U| f\rangle$, when $x$ runs out the set of real numbers, is a Schwartz function. Due to the duality formula (24), we have that

$$
f(x)=\langle x \mid f\rangle=\langle\tilde{x}|U| f\rangle \in \mathcal{S} .
$$

It should be interesting to explore this approach and rewrite Sections II A and II B, where we have used the same notation for the operators independently of the space where they are acting, from this point of view of the representation. However, for the sake of clarity we will continue using the same symbol for any operator $X$ acting on the triplet $\mathcal{S} \subset L^{2}(\mathcal{R}) \subset \mathcal{S}^{\times}$as well as for $U^{-1} X U$ acting on the abstract triplet $\Phi \subset \mathcal{H} \subset \Phi^{\times}$as the existence of such unitary operator $U$ guarantees that the representation triplet and the abstract triplet are isomorphic as shown in (25). Obviously the reader may easily distinguish the actual operator according to the Hilbert space where it acts.

\section{Operators $O(\mathbb{R})$ as $O(\operatorname{UEA}[i o(2)])$}

The great advantage of the rigged Hilbert space (17) is that $\mathcal{S}$ belongs to the domain of all operators in the UEA spanned by $N, a^{\dagger}$, and $a$ and that includes $X$ and $P$. In addition, all operators in this UEA can be extended by duality to operators on $\mathcal{S}^{\times}$, which are continuous under any topology on $\mathcal{S}^{\times}$ compatible with the dual pair.

In $L^{2}(\mathbb{R})$, a complete orthonormal set is formed by the normalized HF $\psi_{n}$, so that we may write $|n\rangle=\left|\psi_{n}\right\rangle \equiv \psi_{n}$ for all $n \in \mathbb{N}$. The action of position $X=\frac{1}{\sqrt{2}}\left(a+a^{\dagger}\right)$, momentum $P=\frac{-i}{\sqrt{2}}\left(a-a^{\dagger}\right)$, number $N=a^{\dagger} a$, and identity $\mathbb{I}$ operators on this basis is

$$
\begin{array}{ll}
X \psi_{n}(x)=x \psi_{n}(x), & P \psi_{n}(x)=-i \frac{d \psi_{n}(x)}{d x}, \\
N \psi_{n}(x)=n \psi_{n}(x), & \mathbb{I} \psi_{n}(x)=\psi_{n}(x) .
\end{array}
$$

Also, the recurrence relations for the Hermite polynomials

$$
\begin{aligned}
& H_{n}^{\prime}(x)=2 n H_{n-1}(x), \\
& H_{n}^{\prime}(x)-2 x H_{n}(x)=H_{n+1}(x),
\end{aligned}
$$


can be rewritten in terms of the operators $a$ and $a^{\dagger}$ and the Hermite functions as:

$$
a \psi_{n}(x)=\sqrt{n} \psi_{n-1}(x), a^{\dagger} \psi_{n}(x)=\sqrt{n+1} \psi_{n}(x),
$$

expressions that coincide with those given in any elementary textbook of quantum mechanics. These relations give the action of $a, a^{\dagger}, X$ and $P$ on the whole $\mathcal{S}$. Furthermore, the extension of $a^{\dagger}$ and $a$ to the dual $\mathcal{S}^{\dagger}$ can be done in an explicit matter in all elements in the UEA. The idea is the following: ${ }^{30}$ let $F \in \mathcal{S}^{\times}$, the sequence of complex numbers given by $\beta_{n}=\left\langle\psi_{n} \mid F\right\rangle$ possesses the following property: the series $\sum_{n=0}^{\infty} \beta_{n} \psi_{n}$ converges to $F$ with the topology on $\mathcal{S}^{\times}$. Since $a$ and $a^{\dagger}$ are continuous on $\mathcal{S}^{\times}$, we obviously have

$$
\begin{aligned}
& a \sum_{n=0}^{\infty} \beta_{n} \psi_{n}=\sum_{n=0}^{\infty} \beta_{n} a \psi_{n}=\sum_{n=0}^{\infty} \beta_{n} \sqrt{n} \psi_{n-1}, \\
& a^{\dagger} \sum_{n=0}^{\infty} \beta_{n} \psi_{n}=\sum_{n=0}^{\infty} \beta_{n} a^{\dagger} \psi_{n}=\sum_{n=0}^{\infty} \beta_{n} \sqrt{n+1} \psi_{n+1} .
\end{aligned}
$$

As is well known, the Lie algebra spanned by $\left\{a, a^{\dagger}, N, \mathbb{I}\right\}$ is isomorphic to the projective algebra $i o(2)$ spanned by $\{N, X, P, \mathbb{I}\}$. The rigged Hilbert space (17) indeed supports a representation with zero value of the Casimir operator $C$ of $i s o(2)^{23}$

$$
\begin{aligned}
C & \equiv \frac{1}{2}\left(X^{2}+P^{2}\right)-\left(N+\frac{1}{2} \mathbb{I}\right) \\
& =\frac{1}{2}\left\{a, a^{\dagger}\right\}-\left(N+\frac{1}{2} \mathbb{I}\right)=0
\end{aligned}
$$

with $\left\{a, a^{\dagger}\right\}$ the anticommutator of $a$ and $a^{\dagger}$, i.e., $\left\{a, a^{\dagger}\right\}=a a^{\dagger}+a^{\dagger} a$. Therefore, for any $n=$ $0,1,2 \ldots$, we have in general

$$
C|n\rangle=\left[\frac{1}{2}\left\{a, a^{\dagger}\right\}-\left(N+\frac{1}{2} \mathbb{I}\right)\right]|n\rangle=0 .
$$

An identical expression comes after replacing $|n\rangle$ by the Hermite function $\psi_{n}(x)$, i.e., $C \psi_{n}(x)=0$ or explicitly

$$
\left[\frac{1}{2}\left(X^{2}-D^{2}\right)-\left(N+\frac{1}{2}\right)\right] \psi_{n}(x)=0,
$$

where $D^{2}=d^{2} / d x^{2}=-P^{2}$. This shows that the group invariant $C$ in (17) is the zero operator on both $\mathcal{S}$ and $\mathcal{S}^{\times}$. In the abstract setting and in the representation supported on (17) we have, respectively,

$$
N=\frac{1}{2}\left(\left\{a, a^{\dagger}\right\}-\mathbb{I}\right), \quad N=\frac{1}{2}\left(X^{2}-D^{2}-\mathbb{I}\right) .
$$

Equation (26) is obviously equivalent to the Hermite differential equation that defines the Hermite polynomials $H_{n}(x)$

$$
H_{n}^{\prime \prime}(x)-2 x H_{n}^{\prime}(x)+2 n H_{n}(x)=0 .
$$

The representation of $U E A[i o(2)]$ on the rigged Hilbert space (17) is irreducible, so that Lie algebra theory confirms that the abstract algebra, spanned by the abstract operators $a, a^{\dagger}, N$, and $\mathbb{I}$, and the representation on (17), spanned by $X, P$ (or $D$ ), $N$, and $\mathbb{I}$ are isomorphic. Thus, each operator $O$ acts on the representation space (17) if and only if it belongs to $U E A[i o(2)]$ and thus can be written (we use the convention of sum over repeated indices)

$$
O=c_{\alpha \beta \gamma} X^{\alpha} D^{\beta} N^{\gamma}=d_{\alpha \beta \gamma} a^{\dagger \alpha} N^{\beta} a^{\gamma} .
$$

From the purely analytical point of view, an ordered monomial of the type $X^{\alpha} D^{\beta} \mathbb{N}^{\gamma} \in U E A$ $[i o(2)]$ is a differential operator of $\beta$ order. But since, from (27), we have

$$
D^{2}=X^{2}-2 N-\mathbb{I},
$$

any operator of $U E A[i o(2)]$ can be reduced to the form

$$
O=f_{0}(X) g_{0}(N)+f_{1}(X) D g_{1}(N),
$$


where $f_{i}(X)$ and $g_{i}(N), i=0,1$, are arbitrary measurable functions. In particular, if we represent these operators on the rigged Hilbert space (17) and apply $O$ to the Hermite functions, we obtain

$$
O \psi_{n}(x)=f_{0}(x) g_{0}(n) \psi_{n}(x)+f_{1}(x) g_{1}(n) \psi_{n}^{\prime}(x),
$$

formula which is completely general.

\section{Substructures obtained from UEA[io(2)]}

The UEA of the Lie algebra $i o(2), U E A[i o(2)]$, is a rich structure and it contains infinitely many $i o(2)$ algebras that allow us to construct an intricate structure of subspaces and operators. Moreover, for any positive integer $k$ we have

$$
\bigoplus_{r=0}^{k-1} i o_{k, r}(2) \subset U E A[i o(2)],
$$

where the pair of integers $(k, r)$ defines a copy $i o_{k, r}(2)$ of the Lie algebra $i o(2)$. As a matter of fact, if we start with $n \in \mathbb{N}$ and $k \neq 0 \in \mathbb{N}$, we can define two other natural numbers, the quotient and the rest, as

$$
\left.\begin{array}{l}
q:=\text { Quotient }[n, k] \\
r:=\operatorname{Mod}[n, k]
\end{array}\right\} \Leftrightarrow n=k q+r,
$$

where $q \in \mathbb{N}$ and $r=0,1,2, \ldots, k-1$. This latter fact, shows that $\{|k q+r\rangle\}$ with $k$ fixed is a complete orthonormal system in the abstract Hilbert space $\mathcal{H}$. In terms of the abstract rigged Hilbert space $\Phi \subset \mathcal{H} \subset \Phi^{\times}$, discussed in Subsection II A, the vectors $|k q+r\rangle$ are in $\Phi$. We can construct two operators $Q$ and $R$ on $\Phi$ as follows:

$$
\begin{aligned}
& Q|k q+r\rangle=q|k q+r\rangle, \\
& R|k q+r\rangle=r|k q+r\rangle .
\end{aligned}
$$

At this point, it is necessary to give some results concerning the construction of relevant RHS for our discussion, which includes properties on the continuity of operators and decompositions arisen after the use of the fractal Fourier transform. This discussion is a bit technical and it is based on standard results given in the bibliography. ${ }^{8,9,14,17,30}$ In particular if $\Phi$ and $\Psi$ are two locally convex vector spaces and $A: \Phi \longmapsto \Psi$ is a linear mapping, then $A$ is continuous if and only if for any seminorm $s$ on $\Psi$, there exists a positive number $K$ and a finite number $s_{1}, s_{2}, \ldots, s_{m}$ of seminorms on $\Phi$, all depending on $s$, such that

$$
s(A \phi) \leq K\left\{s_{1}(\phi)+s_{2}(\phi)+\cdots+s_{m}(\phi)\right\}, \quad \forall \phi \in \Phi .
$$

See, Ref. 30, page 126. Note that, in our case, these seminorms are indeed norms.

Proposition. - The operators $Q$ and $R$ can be extended by linearity and continuity to the spaces $\Phi$ and its dual $\Phi^{\times}$.

Proof. - Since the topology on $\Phi$ is identical to the topology on the Schwartz space $\mathcal{S}$, a vector $\phi$ belongs to $\Phi$ if and only if

$$
\phi=\sum_{n=0}^{\infty} a_{n}|n\rangle
$$

such that

$$
\sum_{n=0}^{\infty}\left|a_{n}\right|^{2}(n+1)^{2 p}<\infty, \quad \forall p \in \mathbb{N} .
$$

Let us consider $N \phi$, i.e.,

$$
N \phi=\sum_{n=0}^{\infty} a_{n} N|n\rangle=\sum_{n=0}^{\infty} a_{n} n|n\rangle .
$$


This operation has a meaning if and only if for all $p=0,1,2 \ldots$ one has

$$
\sum_{n=0}^{\infty}\left|a_{n}\right|^{2} n^{2}(n+1)^{2 p}<\infty .
$$

This is obvious because

$$
\sum_{n=0}^{\infty}\left|a_{n}\right|^{2} n^{2}(n+1)^{2 p} \leq \sum_{n=0}^{\infty}\left|a_{n}\right|^{2}(n+1)^{2 p+2}<\infty .
$$

Since $n=k q+r$, this means that for fixed $k \geq 1$, (29) can be written as

$$
\sum_{r=0}^{\infty} \sum_{q=0}^{k-1}\left|a_{k q+r}\right|^{2}(k q+r)^{2}(k q+r+1)^{2 p}<\infty .
$$

Then, note that

$$
\begin{aligned}
& Q \phi=\sum_{q=0}^{\infty} \sum_{r=0}^{k-1} a_{k q+r} q|k q+r\rangle, \\
& R \phi=\sum_{q=0}^{\infty} \sum_{r=0}^{k-1} a_{k q+r} r|k q+r\rangle .
\end{aligned}
$$

Since $q, r \leq n=k q+r$, one immediately sees that for $p=0,1,2, \ldots$

$$
\begin{aligned}
& \sum_{q=0}^{\infty} \sum_{r=0}^{k-1}\left|a_{k q+r}\right|^{2} q^{2}(k q+r+1)^{2 p}<\infty, \\
& \sum_{q=0}^{\infty} \sum_{r=0}^{k-1}\left|a_{k q+r}\right|^{2} r^{2}(k q+r+1)^{2 p}<\infty,
\end{aligned}
$$

so that the operators $Q$ and $R$ can be extended to the whole $\Phi$. define

The continuity of $Q$ and $R$ can be easily proven. For each $p=0,1,2, \ldots$ and for all $\phi \in \Phi$, we

$$
\|\phi\|_{p}^{2}:=\sum_{q=0}^{\infty} \sum_{r=0}^{k-1}\left|a_{k q+r}\right|^{2}(k q+r+1)^{2 p} .
$$

Then, for all $p=0,1,2, \ldots$, we have

$$
\begin{aligned}
\|Q \phi\|_{p}^{2} & =\sum_{q=0}^{\infty} \sum_{r=0}^{k-1}\left|a_{k q+r}\right|^{2} q^{2}(k q+r+1)^{2 p} \\
& \leq \sum_{q=0}^{\infty} \sum_{r=0}^{k-1}\left|a_{k q+r}\right|^{2}(k q+r+1)^{2 p+2} \\
& =\|\phi\|_{p+1}^{2},
\end{aligned}
$$

and same for $\|R \phi\|_{p}^{2}$. Therefore, using the remark after (28), we conclude that $Q$ and $P$ are continuous on $\Phi$.

The extension of $Q$ and $R$ to the dual $\Phi^{\times}$is defined via a duality formula like in (18). This extension is automatically continuous on $\Phi^{\times}$for any topology compatible with the dual pair $\left(\Phi, \Phi^{\times}\right){ }^{16}$ 
Next, let us consider the operators $A_{k, r}^{\dagger}, A_{k, r} \in U E A[i o(2)]$ defined as

$$
\begin{aligned}
A_{k, r}^{\dagger} & :=\left(a^{\dagger}\right)^{k} \frac{\sqrt{N+k-r}}{\sqrt{k \prod_{j=1}^{k}(N+j)}}, \\
A_{k, r} & :=\frac{\sqrt{N+k-r}}{\sqrt{k \prod_{j=1}^{k}(N+j)}}(a)^{k} .
\end{aligned}
$$

These operators are the formal adjoint of each other and continuous on $\Phi$. Therefore, they can be extended by continuity to the dual $\Phi^{\times}$. On the basis vectors $|k q+r\rangle$ they act as

$$
\begin{aligned}
& A_{k, r}^{\dagger}|k q+r\rangle=\sqrt{q+1}|k(q+1)+r\rangle, \\
& A_{k, r}|k q+r\rangle=\sqrt{q}|k(q-1)+r\rangle .
\end{aligned}
$$

For each pair of integers $k$ and $r$ with $0 \leq r<k$, we have thus an irreducible representation of $i o(2)=$ $\left\langle Q, A_{k, r}^{\dagger}, A_{k, r}, \mathbb{I}\right\rangle$ with $C=0$, denoted by $i o_{k, r}(2)$. The commutators are

$$
\begin{aligned}
{\left[Q, A_{k, r}^{\dagger}\right] } & =+A_{k, r}^{\dagger}, & {\left[Q, A_{k, r}\right] } & =-A_{k, r}, \\
{\left[A_{k, r}, A_{k, r}^{\dagger}\right] } & =\mathbb{I}, & {[\mathbb{I}, \cdot] } & =0,
\end{aligned}
$$

where $\cdot$ denotes an arbitrary algebra element. This shows that for $k$ and $r$ fix, we are not considering neither the whole space $\Phi$ nor the whole Hilbert space $L^{2}(\mathbb{R})$, but closed subspaces of them. Take, for instance, $k=4$. Then, $0 \leq r<4$. For each value of $r=0,1,2,3$, let us consider the set of orthonormal vectors $|4 q+r\rangle$, with $q \in \mathbb{N}$. These vectors span a closed subspace, $\mathcal{H}_{4, r}$ of $\mathcal{H}$. Obviously,

$$
\mathcal{H}=\bigoplus_{r=0}^{3} \mathcal{H}_{4, r} .
$$

In terms of the representation of $\mathcal{H}$ by $L^{2}(\mathbb{R})$ and recalling that the vectors $|4 q+r\rangle$ admit a representation by the normalized Hermite functions $\psi_{4 q+r}(x), q=0,1,2, \ldots$, we are considering the closed subspace $L_{4, r}^{2}(\mathbb{R})$ of $L^{2}(\mathbb{R})$ spanned by these functions for fixed value of $r=0,1,2,3$. As in (30), one clearly has

$$
L^{2}(\mathbb{R})=\bigoplus_{r=0}^{3} L_{4, r}^{2}(\mathbb{R}) .
$$

In consequence, the algebra of operators on $\mathcal{H}_{4, r}$ and on $L_{4, r}^{2}(\mathbb{R})$ that we are considering is isomorphic to the $U E A\left[i o_{4, r}(2)\right]$.

Although this is just an illustrative example, these results are indeed general, simply by replacing 4 by an arbitrary positive integer $k$ and letting $r$ go from 0 to $k-1$. Thus,

$$
\mathcal{H}=\bigoplus_{r=0}^{k-1} \mathcal{H}_{k, r}, \quad L^{2}(\mathbb{R})=\bigoplus_{r=0}^{k-1} L_{k, r}^{2}(\mathbb{R}),
$$

$\forall k \in \mathbb{N}-\{0\}$ and $O \in U E A\left[i o_{k, r}(2)\right]$ iff

$$
O|k q+r\rangle=\sum_{q^{\prime}} c_{q^{\prime}}\left|k q^{\prime}+r\right\rangle .
$$

The spaces $\Phi_{k, r}$ and $\mathcal{S}_{k, r}$ can be easily obtained. A vector $\phi$ belongs to $\Phi_{k, r}$ if and only if

$$
\phi=\sum_{q=0}^{\infty} a_{q}|k q+r\rangle
$$

with 


$$
\sum_{q=0}^{\infty}\left|a_{q}\right|^{2}(q+1)^{2 p}<\infty, \quad p=0,1,2, \ldots
$$

The same result can be achieved for any function in $\mathcal{S}_{k, r}$, just replacing $|k q+r\rangle$ in (32) by the normalized Hermite functions $\psi_{k q+r}(x)$. We obtain thus the rigged Hilbert spaces

$$
\begin{aligned}
& \Phi_{k, r} \subset \mathcal{H}_{k, r} \subset \Phi_{k, r}^{\times}, \\
& \mathcal{S}_{k, r} \subset L_{k, r}^{2}(\mathbb{R}) \subset \mathcal{S}_{k, r}^{\times} .
\end{aligned}
$$

In conclusion, there are infinitely many decompositions of $R H S(\mathbb{R})$, one for each $k \in \mathbb{N}-\{0\}$, in rigged Hilbert subspaces. Each decomposition contains $k$ subspaces, each of them labeled by the pair $(k, r)$. Besides $O \in U E A[i o(2)]$ if and only if $O$ is an operator of $\mathcal{H}$, while $O \in U E A\left[i o_{k, r}(2)\right]$ iff $O$ is an operator of $\mathcal{H}_{k, r}$.

\section{E. Subspaces obtained from fractional Fourier transform}

It is quite interesting to note that the split of $L^{2}(\mathbb{R})$ given by (31) has a deep connection with the so called fractional Fourier transform. This is a generalization of the Fourier transform. It admits several equivalent definitions, ${ }^{6}$ although the simplest one can be introduced via the decomposition of a function in $L^{2}(\mathbb{R})$ in terms of the normalized Hermite functions $\psi_{n}(x)(2)$.

Let us denote the fractional Fourier transform of $f \in L^{2}(\mathbb{R})$ associated to $a \in \mathbb{R}$ by $\mathcal{F}^{a} f$. Since

$$
f(x)=\sum_{n=0}^{\infty} a_{n} \psi_{n}(x)
$$

with

$$
a_{n}=\int_{-\infty}^{\infty} f^{*}(x) \psi_{n}(x) d x
$$

we define $\mathcal{F}^{a} f$ in terms of the decomposition (34) as

$$
\left[\mathcal{F}^{a} f\right](p):=\sum_{n=0}^{\infty} a_{n} e^{i n a \pi / 2} \psi_{n}(p)
$$

Series in (35) obviously converges in both the $L^{2}(\mathbb{R})$ norm and in the sense given in (32) provided that $f(x) \in \mathcal{S}$, so that $\mathcal{F}^{a} f \in \mathcal{S}$ if $f \in \mathcal{S}$. Now we restrict to $a=4 / k$ with $k$ a positive integer (the Fourier transform corresponds to $k=4$, i.e., $a=1$ ). Thereafter we write

$$
\begin{aligned}
\tilde{f}^{k}(p) & :=\left[\mathcal{F}^{4 / k} f\right](p) \\
& =\sum_{n=0}^{\infty} a_{n} e^{2 \pi i n / k} \psi_{n}(p) .
\end{aligned}
$$

Then, for each $k \in \mathbb{N}-\{0\}$ the set of natural numbers can be split into $k$ disjoint sequences where $q$ varies from 0 to $\infty$ as follows $(n=k q+r)$ :

$$
\{k q\},\{k q+1\}, \ldots,\{k q+k-1\} .
$$


Note on the relation between the numbers $k q+r, r=0,1,2, \ldots, k-1$ and the $k$-th roots of unity (see expression (36)). With this idea in mind we can split $\tilde{f}^{k}$ as

$$
\begin{gathered}
\tilde{f}^{k}(p)=\sum_{q=0}^{\infty} a_{k q} e^{2 \pi(k q) i / k} \psi_{k q}(p)+\sum_{q=0}^{\infty} a_{k q+1} e^{2 \pi(k q+1) i / k} \psi_{k q+1}(p) \\
+\cdots+\sum_{q=0}^{\infty} a_{k q+k-1} e^{2 \pi(k q+k-1) i / k} \psi_{k q+k-1}(p) \\
=\sum_{q=0}^{\infty} a_{k q} \psi_{k q}(p)+e^{2 \pi i / k} \sum_{q=0}^{\infty} a_{k q+1} \psi_{k q+1}(p) \\
+\cdots+e^{2 \pi(k q+k-1) i / k} \sum_{q=0}^{\infty} a_{k q+k-1} \psi_{k q+k-1}(p) \\
=f_{0}^{k}(p)+e^{2 \pi i / k} f_{1}^{k}(p)+\cdots+e^{2 \pi(k q+k-1) i / k} f_{k-1}^{k}(p) \\
=\tilde{f}_{0}^{k}(p)+\tilde{f}_{1}^{k}(p)+\cdots+\tilde{f}_{k-1}^{k}(p),
\end{gathered}
$$

where

$$
\begin{aligned}
f_{r}^{k}(x) & :=\sum_{q=0}^{\infty} a_{k q+r} \psi_{k q+r}(x), \\
\tilde{f}_{r}^{k}(p) & :=e^{2 \pi r i / k} f_{r}^{k}(p),
\end{aligned}
$$

and $r=0,1, \ldots, k-1$. The vectors $\tilde{f}_{r}^{k},(r=0,1, \ldots, k-1)$ are mutually orthogonal, so that (37) gives a split of $L^{2}(\mathbb{R})$ into an orthonormal direct sum of subspaces. Furthermore, having in mind that $\tilde{f}_{r}^{k}(p) \equiv\left[\mathcal{F}^{4 / k} f_{r}^{k}\right](p)$, we see that each term in the direct sum is an eigen-subspace of $\mathcal{F}^{4 / k}$ with eigenvalue $e^{i 2 \pi r / k}$, i.e., independently from the bases $\{|x\rangle\}$ or $\{|p\rangle\}$

$$
L^{2}(\mathbb{R})=L_{k, 0}^{2}(\mathbb{R}) \oplus L_{k, 1}^{2}(\mathbb{R}) \oplus \cdots \oplus L_{k, k-1}^{2}(\mathbb{R}) .
$$

In addition, $f_{r}^{k}$ as in (37) has the property that

$$
\sum_{q=0}^{\infty}\left|a_{k q+r}\right|^{2}(k q+r)^{2 p}<\infty, \quad p \in \mathbb{N}
$$

then, $f_{r}^{k} \in \mathcal{S}_{k, r}$. Thus, for each $k$, the rigged Hilbert space $\mathcal{S} \subset L^{2}(\mathbb{R}) \subset \mathcal{S}^{\times}$admits a split into $k$ rigged Hilbert spaces

$$
\mathcal{S}_{k, r} \subset L_{k, r}^{2}(\mathbb{R}) \subset \mathcal{S}_{k, r}^{\times}
$$

with

$$
\mathcal{S}=\bigoplus_{r=0}^{k-1} \mathcal{S}_{k, r}
$$

This induces a similar splitting in the abstract rigged Hilbert space $\Phi \subset \mathcal{H} \subset \Phi^{\times}$, for which $\mathcal{S} \subset L^{2}(\mathbb{R}) \subset \mathcal{S}^{\times}$is a representation such that $U \mathcal{H}=L^{2}(\mathbb{R})$. This splitting is given by

$$
\Phi_{k, r} \subset \mathcal{H}_{k, r} \subset \Phi_{k, r}^{\times},
$$

with $U \mathcal{H}_{k, r}=L_{k, r}^{2}(\mathbb{R})$ and $U \Phi_{k, r}=\mathcal{S}_{k, r}$.

Note that the RHS structure displayed in (33) has been obtained again but this time without any reference to the operator space. 


\section{WAVE FUNCTIONS AND SIGNALS ON $\mathbb{R}^{+}$}

\section{A. Rigged Hilbert space on $\mathbb{R}^{+}$and Laguerre functions}

Let us consider the space of square integrable functions on $\mathbb{R}^{+} \equiv[0,+\infty)$, denoted by $L^{2}\left(\mathbb{R}^{+}\right)$. A complete orthonormal set in $L^{2}\left(\mathbb{R}^{+}\right)$is, with $\alpha \in(-1,+\infty)$ fix,

$$
M_{n}^{\alpha}(y)=\sqrt{\frac{\Gamma(n+1)}{\Gamma(n+\alpha+1)}} y^{\alpha / 2} e^{-y / 2} L_{n}^{\alpha}(y),
$$

where $n=0,1,2, \ldots$, and $L_{n}^{\alpha}(y)$ are the associated Laguerre polynomials. ${ }^{31,32}$ The expressions of orthonormality and completeness that hold for these functions are

$$
\begin{aligned}
& \int_{0}^{\infty} M_{n}^{\alpha}(y) M_{m}^{\alpha}(y) d y=\delta_{n m}, \\
& \sum_{n=0}^{\infty} M_{n}^{\alpha}(y) M_{n}^{\alpha}\left(y^{\prime}\right)=\delta\left(y-y^{\prime}\right) .
\end{aligned}
$$

In order to construct a rigged Hilbert space, let us consider this orthonormal basis and let us choose in $L^{2}\left(\mathbb{R}^{+}\right)$the space of all functions

$$
f(y)=\sum_{n=0}^{\infty} a_{n} M_{n}^{\alpha}(y)
$$

such that

$$
\sum_{n=0}^{\infty}\left|a_{n}\right|^{2} n^{2 p}(n+\alpha)^{2 p}<\infty, \quad p=0,1,2, \ldots .
$$

We shall denote this space by $\mathcal{D}_{\alpha}$. The space $\mathcal{D}_{\alpha}$ is a nuclear subspace dense in $L^{2}\left(\mathbb{R}^{+}\right)$, so that $\mathcal{D}_{\alpha} \subset L^{2}\left(\mathbb{R}^{+}\right) \subset \mathcal{D}_{\alpha}^{\times}$is a rigged Hilbert space. Furthermore, $\mathcal{D}_{\alpha}$ is a countably normed space with norms

$$
\|f(y)\|_{p}=\sqrt{\sum_{n=0}^{\infty}\left|a_{n}\right|^{2} n^{2 p}(n+\alpha)^{2 p},}
$$

with $p=0,1,2, \ldots$, and for any $f(y) \in \mathcal{D}_{\alpha}$.

Next, we consider the following operators on $L^{2}\left(\mathbb{R}^{+}\right)$:

$$
\begin{array}{ll}
Y M_{n}^{\alpha}(y):=y M_{n}^{\alpha}(y), & D_{y} M_{n}^{\alpha}(y):=M_{n}^{\alpha}(y)^{\prime}, \\
N M_{n}^{\alpha}(y):=n M_{n}^{\alpha}(y), & \mathbb{I} M_{n}^{\alpha}(y):=M_{n}^{\alpha}(y) .
\end{array}
$$

Note that $Y$ is the multiplication operator, $D_{y}$ is the derivation by $y, N$ is a number operator, and $\mathbb{I}$ is the identity. We need to show that $Y$ reduces $\mathcal{D}_{\alpha}$, which means that $Y g(y) \in \mathcal{D}_{\alpha}$ for any $g(y) \in \mathcal{D}_{\alpha}$, that $Y$ is essentially self-adjoint with domain $\mathcal{D}_{\alpha}$ and that $Y$ is continuous with the above topology on $\mathcal{D}_{\alpha}$. To do it, let us consider the following operators:

$$
\begin{aligned}
& J_{+}:=\left(Y D_{y}+N+1+\frac{\alpha-Y}{2}\right), \\
& J_{-}:=\left(-Y D_{y}+N+\frac{\alpha-Y}{2}\right) .
\end{aligned}
$$

The action of $J_{ \pm}$on the basis functions $M_{n}^{\alpha}(y)$ is

$$
\begin{aligned}
& J_{+} M_{n}^{\alpha}(y)=\sqrt{(n+1)(n+\alpha+1)} M_{n+1}^{\alpha}(y) \\
& J_{-} M_{n}^{\alpha}(y)=\sqrt{n(n+\alpha)} M_{n-1}^{\alpha}(y) .
\end{aligned}
$$

From (43) we obtain the following expression for $Y$ :

$$
Y=-\left(J_{+}+J_{-}\right)+2 N+(\alpha+1) \mathbb{I} .
$$


Proposition. (1) $Y$ with domain $\mathcal{D}_{\alpha}$ is essentially selfadjoint on $L^{2}\left(\mathbb{R}^{+}\right)$. (2) $\mathcal{D}_{\alpha}$ reduces $Y$ and $Y$ is a continuous operator on $\mathcal{D}_{\alpha}$. (3) $Y$ has a continuous spectrum $\sigma(Y) \equiv \mathbb{R}$.

Proof. (1) First of all, note that $Y$ is symmetric (Hermitian), since $J_{ \pm}$are Hermitian conjugate of each other. The functions $M_{n}^{\alpha}(y)$ are in the range of the operators $Y \pm i I$, so that both ranges are dense. (2) For any $p=0,1,2, \ldots$ and any $f(y) \in \mathcal{D}_{\alpha}$, it is a simple exercise to show that $\|Y f\|_{p} \leq 3\|f\|_{p+1}+|\alpha-1|\|f\|_{p}$, where $\|-\|_{p}$ is defined as in (42). (3) The proof goes as the operator $X$ for the harmonic oscillator.

The Gelfand triplet $\mathcal{D}_{\alpha} \subset L^{2}\left(\mathbb{R}^{+}\right) \subset \mathcal{D}_{\alpha}^{\times}$serves as a representation of an abstract setting. Let $\mathcal{H}$ be an arbitrary infinite dimensional separable Hilbert space. We know that $\mathcal{H}$ and $L^{2}\left(\mathbb{R}^{+}\right)$ are unitarily equivalent. This implies the existence of unitary operators $U: \mathcal{H} \longmapsto L^{2}\left(\mathbb{R}^{+}\right)$. The non-uniqueness of $U$ is not a problem, choose any one. Then, define $\Phi_{\alpha}:=U^{-1} \mathcal{D}_{\alpha}$ and transport the topology on $\mathcal{D}_{\alpha}$ to $\Phi_{\alpha}$ by $U^{-1}$. We have a new rigged Hilbert space $\Phi_{\alpha} \subset \mathcal{H} \subset \Phi_{\alpha}^{\times}$. Hence $U^{-1} Y U$ on $\Phi_{\alpha} \subset \mathcal{H} \subset \Phi_{\alpha}^{\times}$has the same properties than $Y$ on $\mathcal{D}_{\alpha} \subset L^{2}\left(\mathbb{R}^{+}\right) \subset \mathcal{D}_{\alpha}^{\times}$and, in particular, the same spectral properties. After the Gelfand-Maurin theorem, there exists a set of generalized eigenvectors of $U^{-1} Y U,|y\rangle$, where $y$ runs out $\mathbb{R}^{+}$such that (we omit the index $\alpha$ and we use the same symbol for $Y$ as well as $U^{-1} Y U$-see last paragraph of Subsection II B - for simplicity in the notation)

$$
\begin{aligned}
& Y|y\rangle=y|y\rangle,\left\langle y \mid y^{\prime}\right\rangle=\delta\left(y-y^{\prime}\right), \\
& \int_{-\infty}^{\infty}|y\rangle\langle y| d y=\mathbb{I},
\end{aligned}
$$

where $\mathbb{I}$ is the identity $\mathbb{I}: \Phi_{\alpha} \longmapsto \mathcal{H}$. For any fixed $n \in \mathbb{N}$ and $\alpha \in(-1,+\infty)$, let us define the vector $|n\rangle$ as

$$
|n\rangle:=\int_{0}^{\infty} d y M_{n}^{\alpha}(y)|y\rangle .
$$

Properties (40) and (46) yield to the following important properties of vectors $|n\rangle$ :

$$
\langle n \mid m\rangle=\delta_{n m}, \quad \sum_{n=0}^{\infty}|n\rangle\langle n|=\mathbb{I} .
$$

This shows that $|n\rangle$ with $n \in \mathbb{N}$ is a complete orthonormal set (orthonormal basis) in $\mathcal{H}$. As a matter of fact, $U|n\rangle=M_{n}^{\alpha}$, once $\alpha>-1$ has been fixed. For $y \geq 0$, we have that

$$
\begin{aligned}
\langle y \mid n\rangle & =\int_{0}^{\infty} d y^{\prime} M_{n}^{\alpha}\left(y^{\prime}\right)\left\langle y \mid y^{\prime}\right\rangle \\
& =\int_{0}^{\infty} d y^{\prime} M_{n}^{\alpha}\left(y^{\prime}\right) \delta\left(y-y^{\prime}\right) \\
& =M_{n}^{\alpha}(y) .
\end{aligned}
$$

This suggests that for $y<0$, we should define $\langle y \mid n\rangle=0$. Then, we have returned to a situation that have showed up in the Section II, i.e., the presence of two different bases for the elements in $\Phi_{\alpha}$ : a continuous basis $\left\{|y\rangle, y \in \mathbb{R}^{+}\right\}$and a discrete basis $\{|n\rangle, n \in \mathbb{N}\}$, so that for any $|f\rangle \in \Phi_{\alpha}$, we have

$$
\begin{aligned}
& |f\rangle=\mathbb{I}|f\rangle=\left(\int_{-\infty}^{\infty} d y|y\rangle\langle y|\right)|f\rangle=\int_{-\infty}^{\infty} d y f(y)|y\rangle, \\
& |f\rangle=\left(\sum_{n=0}^{\infty}|n\rangle\langle n|\right)|f\rangle=\sum_{n=0}^{\infty}|n\rangle\langle n \mid f\rangle=\sum_{n=0}^{\infty} f_{n}|n\rangle,
\end{aligned}
$$

where $f(y)=\langle y \mid f\rangle$ and $f_{n}=\langle n \mid f\rangle$. In addition and more important:

$$
\begin{aligned}
f(y) & =\langle y \mid f\rangle=\left\langle y\left|\left(\sum_{n=0}^{\infty}|n\rangle\langle n|\right)\right| f\right\rangle \\
& =\sum_{n=0}^{\infty}\langle y \mid n\rangle\langle n \mid f\rangle=\sum_{n=0}^{\infty} M_{n}^{\alpha}(y) f_{n},
\end{aligned}
$$


and

$$
\begin{aligned}
f_{n} & =\langle n \mid f\rangle=\left\langle n\left|\left(\int_{-\infty}^{\infty}|y\rangle\langle y| d y\right)\right| f\right\rangle \\
& =\int_{0}^{\infty}\langle n \mid y\rangle\langle y \mid f\rangle d y=\int_{0}^{\infty} M_{n}^{\alpha}(y) f(y) d y .
\end{aligned}
$$

In the second identity in this last expression, we have used that $\langle n \mid y\rangle=\langle y \mid n\rangle=0$ if $y<0$. Thus, the wave function $f(y)$ and the sequence $\left\{f_{n}\right\}$ describe equally well the vector state $|f\rangle$, which can be spanned in terms of either the continuous $\{|y\rangle\}$ or the discrete $\{|n\rangle\}$ basis. On the other hand, a vector $|f\rangle \in \mathcal{H}$ belongs to $\Phi_{\alpha}$ if and only if $\sum_{n=0}^{\infty}\left|f_{n}\right|^{2}(n+1)^{2 n}<\infty$.

We define the operator $N$ on the basis $|n\rangle$ as $N|n\rangle=n|n\rangle$ and the operator $U N U^{-1}$ (also denoted $N$ ) on $\mathcal{D}_{\alpha}$, so that $N M_{n}^{\alpha}(y)=n M_{n}^{\alpha}(y)$. The operator $N$ is extended to $\Phi_{\alpha}$ by linearity and continuity, same $N$ to $\mathcal{D}_{\alpha}$. By the duality formula these operators can be extended to $\Phi_{\alpha}^{\times}$and $\mathcal{D}_{\alpha}{ }^{\times}$, respectively.

\section{B. The algebra of operators on $\mathbb{R}^{+}$}

Let us come back to the operators $J_{ \pm}$defined in (43). Because of (44) these operators can be extended to all $\mathcal{D}_{\alpha}$ by linearity and continuity. Since $\left\|J_{ \pm} f\right\|_{p} \leq\|f\|_{p+1}$ for any $f(y) \in \mathcal{D}_{\alpha}$, these operators are continuous on $\mathcal{D}_{\alpha}$. In addition, $J_{\mp}$ is the Hermitian conjugate of the operator $J_{ \pm}$. Unlike the previous case, in order to define these rising and lowering operators we now need the number operator $N$. Booth operators $J_{ \pm}$together with the extension on $\mathcal{D}_{\alpha}$ by linearity and continuity of the operator

$$
\begin{aligned}
J_{3} & :=N+\frac{\alpha+1}{2} \mathbb{I}, \\
J_{3} M_{n}^{\alpha}(y) & :=\left(n+\frac{\alpha+1}{2}\right) M_{n}^{\alpha}(y),
\end{aligned}
$$

close the Lie algebra $s u(1,1)$ since the commutation relations between these operators are

$$
\left[J_{3}, J_{ \pm}\right]= \pm J_{ \pm}, \quad\left[J_{+}, J_{-}\right]=-2 J_{3} .
$$

Note that all the elements of the algebra are continuous operators on $\mathcal{D}_{\alpha}$ (and hence, they admit continuous extensions to the dual $\mathcal{D}_{\alpha}^{\times}$). A similar construction has been discussed in Ref. 33 .

This discussion can be brought into $\Phi_{\alpha}$, where the operators $J_{ \pm}$and $J_{3}$ should be replaced by $U J_{ \pm} U^{-1}$ and $U J_{3} U^{-1}$, also denoted $J_{ \pm}$and $J_{3}$, respectively. Then, one obviously has

$$
\begin{aligned}
& J_{+}|n\rangle=\sqrt{(n+1)(n+\alpha+1)}|n+1\rangle, \\
& J_{3}|n\rangle=(n+(\alpha+1) / 2)|n\rangle, \\
& J_{-}|n\rangle=\sqrt{n(n+\alpha)}|n-1\rangle .
\end{aligned}
$$

These operators are continuous on $\Phi_{\alpha}$ and continuously extensible to the dual $\Phi_{\alpha}^{\times}$. The Casimir operator $C$ of $s u(1,1)$ is

$$
C=J_{3}^{2}-\frac{1}{2}\left\{J_{+}, J_{-}\right\}=\frac{\alpha^{2}-1}{4} \mathbb{I} .
$$

Obviously, the Casimir operator for the representation of the $s u(1,1)$ Lie algebra on $\mathcal{D}_{\alpha}, C$, is the image of (55) by $U$, so it has the same formal expression of $C$. Using (43), (44), and (50), we can transform the Casimir into

$$
\left[Y D_{y}^{2}+D_{y}+\mathbb{N}+\frac{\alpha+1}{2} \mathbb{I}-\frac{\alpha^{2}}{2 Y}-\frac{Y}{4}\right] M_{n}^{\alpha}(y)=0,
$$

for all $n=0,1,2 \ldots$, where $Y^{-1} M_{n}^{\alpha}(y)=y^{-1} M_{n}^{\alpha}(y)$. This equation is the operator equivalent to the associated Laguerre equation,

$$
y L_{n}^{\alpha}(y)^{\prime \prime}+(\alpha+1-y) L_{n}^{\alpha}(y)^{\prime}+n L_{n}^{\alpha}(y)=0 .
$$


Note that the operator

$$
Y\left(Y D_{y}^{2}+D_{y}-\frac{\alpha^{2}}{2 Y}\right)
$$

is continuous on $\mathcal{D}_{\alpha}$. In any case, on $\mathcal{D}_{\alpha}$ and its dual $\mathcal{D}_{\alpha}^{\times}$, we have the following operational identity:

$$
N=-Y D_{y}^{2}-D_{y}-\frac{\alpha+1}{2} \mathbb{I}+\frac{\alpha^{2}}{2 Y}+\frac{Y}{4} .
$$

This representation of $s u(1,1)$ is irreducible. The universal enveloping algebra of $s u(1,1), U E A$ $[s u(1,1)]$, is given by all the operators of the following form:

$$
O=\sum c_{\alpha \beta \gamma} J_{+}^{\alpha} J_{3}^{\beta} J_{-}^{\gamma}=\sum d_{\alpha \beta \gamma} Y^{\alpha} D_{y}^{\beta} N^{\gamma} .
$$

From the first identity on this expression, we note that operators in the enveloping algebra $U E A$ [su(1,1)] must be continuous on $\mathcal{D}_{\alpha}$. While $\alpha, \beta$, and $\gamma$ in the first sum are independent, this cannot be the case in the second sum, since $Y$ and $D_{y}$ do not even leave invariant $\mathcal{D}_{\alpha}$. The continuity of a monomial of the form $Y^{\alpha} D_{y}^{\beta}$ demands that $\alpha \geq \beta$. It is also clear that the rigged Hilbert space $\Phi_{\alpha} \subset \mathcal{H} \subset \Phi_{\alpha}^{\times}$supports an equivalent representation of $U E A[\operatorname{su}(1,1)]$.

Finally, it is important to remark that a decomposition like (31) is also possible here and takes the following form for all positive integer $k$ :

$$
\mathcal{H}=\bigoplus_{r=0}^{k-1} \mathcal{H}_{k, r}, \quad L^{2}\left(\mathbb{R}^{+}\right)=\bigoplus_{r=0}^{k-1} L_{k, r}^{2}\left(\mathbb{R}^{+}\right) .
$$

This split is made by complete analogy with the construction valid in the previous case.

\section{Fourier-like transformations supported on $\mathbb{R}^{+}$}

In Subsection II E, we have studied the fractional Fourier transform associated to the Hermite functions. The basic idea of the construction there was that the Hermite functions are eigenfunctions of the ordinary Fourier transform. We would have liked to extend that formalism to the generalized Laguerre functions. This is not possible as $M_{n}^{\alpha}(y)$ are not eigenfunctions of the Fourier transform. Nevertheless, we may try to deal with this problem replacing the Fourier transform by some related integral transform. In fact, there exist at least two of these type of transforms for which a decomposition in the spirit of Subsection II E can be done for two exceptional cases corresponding to the values $\alpha= \pm 1 / 2$. Taking into account the relations between Hermite and Laguerre polynomials,

$$
\begin{aligned}
H_{2 n}(x) & =(-1)^{n} 2^{2 n} n ! L_{n}^{-1 / 2}\left(x^{2}\right), \\
H_{2 n+1}(x) & =(-1)^{n} 2^{2 n+1} n ! x L_{n}^{+1 / 2}\left(x^{2}\right),
\end{aligned}
$$

we get that

$$
\begin{aligned}
& \psi_{2 n}(x)=(-1)^{n}\left(x^{2}\right)^{1 / 4} M_{n}^{-1 / 2}\left(x^{2}\right), \\
& \psi_{2 n+1}(x)=(-1)^{n} x\left(x^{2}\right)^{-1 / 4} M_{n}^{1 / 2}\left(x^{2}\right) .
\end{aligned}
$$

Hence, we are on the position of defining the transforms $\mathcal{T}_{ \pm}$on functions $f(y) \in L^{2}\left(\mathbb{R}^{+}\right)$as follows:

$$
\begin{aligned}
& {\left[\mathcal{T}_{+} f\right](s):=\frac{1}{\sqrt{2 \pi}} \int_{0}^{\infty} d y \frac{\sin [\sqrt{s y}]}{(s y)^{1 / 4}} f(y),} \\
& {\left[\mathcal{T}_{-} f\right](s):=\frac{1}{\sqrt{2 \pi}} \int_{0}^{\infty} d y \frac{\cos [\sqrt{s y}]}{(s y)^{1 / 4}} f(y) .}
\end{aligned}
$$

These transforms verify the following relations:

$$
\left[\mathcal{T}_{ \pm} M_{n}^{ \pm 1 / 2}\right](s)=(-1)^{n} M_{n}^{ \pm 1 / 2}(s),
$$


so that $M_{n}^{ \pm 1 / 2}(s)$ are eigenfunctions of the operators $\mathcal{T}_{ \pm}$, respectively, with eigenvalues $(-1)^{n}$. Thus, if

$$
f(y)=\sum_{n=0}^{\infty} a_{n}^{ \pm} M_{n}^{ \pm 1 / 2}(y),
$$

with

$$
a_{n}^{ \pm}=\int_{0}^{\infty} f^{*}(y) M_{n}^{ \pm 1 / 2}(y) d y
$$

we can define two new fractional integral transforms for each $a \in \mathbb{R}, \mathcal{T}_{ \pm}^{a}$, by

$$
\left[\mathcal{T}_{ \pm}^{a} f\right](s):=\sum_{n=0}^{\infty} a_{n}^{ \pm} e^{i n a \pi / 2} M_{n}^{ \pm 1 / 2}(s) .
$$

Then for $a=4 / k$ with $k \in \mathbb{N}-\{0\}$ we have

$$
\begin{gathered}
\tilde{f}_{ \pm}^{k}(s):=\left[\mathcal{T}_{ \pm}^{4 / k} f\right](s)=\sum_{q=0}^{\infty} a_{k q}^{ \pm} e^{-2 \pi(k q) i / k} M_{k q}^{ \pm 1 / 2}(s)+\sum_{q=0}^{\infty} a_{k q+1}^{ \pm} e^{-2 \pi(k q+1) i / k} M_{k q+1}^{ \pm 1 / 2}(s)+\cdots \\
\ldots+\sum_{q=0}^{\infty} a_{k q+k-1}^{ \pm} e^{-2 \pi(k q+k-1) i / k} M_{k q+k-1}^{ \pm 1 / 2}(s) \\
=\sum_{q=0}^{\infty} a_{k q}^{ \pm} M_{k q}^{ \pm 1 / 2}(s)+e^{-2 \pi i / k} \sum_{q=0}^{\infty} a_{k q+1}^{ \pm} M_{k q+1}^{ \pm 1 / 2}(s)+\cdots \\
+e^{-2 \pi(k-1) i / k} \sum_{q=0}^{\infty} a_{k q+k-1}^{ \pm} M_{k q+k-1}^{ \pm 1 / 2}(s) \\
=f_{0, \pm}^{k}(s)+e^{-2 \pi i / k} f_{1, \pm}^{k}(s)+\cdots+e^{-2 \pi(k-1) i / k} f_{k-1, \pm}^{k}(s),
\end{gathered}
$$

where

$$
f_{r, \pm}^{k}(s):=\sum_{q=0}^{\infty} a_{k q+r}^{ \pm} M_{k q+r}^{ \pm 1 / 2}(s) .
$$

So that for each $k \in \mathbb{N}-\{0\}$ we have again the decomposition (56) of $L^{2}\left(\mathbb{R}^{+}\right)$

$$
\begin{aligned}
& L^{2}\left(\mathbb{R}^{+}\right)=\bigoplus_{r=0}^{k-1} L_{k, r}^{2}\left(\mathbb{R}^{+}\right)^{ \pm} \\
& =L_{k, 0}^{2}\left(\mathbb{R}^{+}\right)^{ \pm} \oplus L_{k, 1}^{2}\left(\mathbb{R}^{+}\right)^{ \pm} \oplus \cdots \oplus L_{k, k-1}^{2}\left(\mathbb{R}^{+}\right)^{ \pm},
\end{aligned}
$$

where each of the closed subspaces $L_{k, r}^{2}\left(\mathbb{R}^{+}\right)^{ \pm}$is an eigen-subspace of $\mathcal{T}_{ \pm}$with eigenvalue $e^{-2 \pi r i / k}$. Obviously, like in (56) each $L_{k, r}^{2}\left(\mathbb{R}^{+}\right)^{ \pm}$is spanned by the complete orthonormal set $\left\{M_{k q+r}^{ \pm 1 / 2}(y) \mid\right.$ $q \in \mathbb{N}\}$.

Let us go back to the rigged Hilbert space $\mathcal{D}_{\alpha} \subset L^{2}\left(\mathbb{R}^{+}\right) \subset \mathcal{D}_{\alpha}^{\times}$. In our case, $\alpha= \pm 1 / 2$ so that we shall henceforth use the notation $\mathcal{D}_{ \pm} \subset L^{2}\left(\mathbb{R}^{+}\right) \subset \mathcal{D}_{ \pm}^{\times}$to denote this pair of RHS. Then, fixed $k$, the decomposition of $L^{2}\left(\mathbb{R}^{+}\right)=\oplus_{r=0}^{k-1} L_{k, r}^{2}\left(\mathbb{R}^{+}\right)^{ \pm}$induces on $\mathcal{D}_{ \pm}$the following decomposition as an orthogonal sum of subspaces: $\mathcal{D}_{ \pm}:=\oplus_{r=0}^{k-1} \mathcal{D}_{k, r}^{ \pm}$. For each pair $(k, r)$, the triplet $\mathcal{D}_{k, r}^{ \pm} \subset L_{k, r}^{2}\left(\mathbb{R}^{+}\right)^{ \pm} \subset$ $\left(\mathcal{D}_{k, r}^{ \pm}\right)^{\times}$is a RHS. A similar decomposition can be performed on the abstract triplet $\Phi_{ \pm} \subset \mathcal{H} \subset \Phi_{ \pm}^{\times}$. The procedure goes as in the previous case (see Subsection II E).

From the definition of $\mathcal{T}^{ \pm}$, we can conclude that these operators leave invariant and are continuous on all $\mathcal{D}_{k, r}^{ \pm}$. This means that $\mathcal{T}^{ \pm}$can be continuously extended into the duals and also that they induce transformations with the same properties on $\Phi_{k, r}^{ \pm}$. These are $U \mathcal{T}^{ \pm} U^{-1}$, where $U$ is a unitary transformation $U \mathcal{H}=L^{2}\left(\mathbb{R}^{+}\right)$.

The operator $Y$ defined in (3.10) is reduced by the spaces $\mathcal{D}_{ \pm}$and, therefore, can be extended into the duals $\mathcal{D}_{ \pm}^{\times}$by the duality formula. Thus, the Gelfand-Maurin theorem provides of two complete generalized bases $\left|y^{ \pm}\right\rangle \in \mathcal{D}_{ \pm}^{\times}$such that $Y\left|y^{ \pm}\right\rangle=y\left|y^{ \pm}\right\rangle$. Dual spaces $\mathcal{D}_{ \pm}^{\times}$are naturally isomorphic. This isomorphism transforms the continuous basis $\left\{\left|y^{+}\right\rangle\right\}$into the continuous basis $\left\{\left|y^{-}\right\rangle\right\}$ 
and allows us to identify $\left|y^{+}\right\rangle$and $\left|y^{-}\right\rangle$by an abuse of language whenever necessary. However

$$
\begin{aligned}
& \left|s^{+}\right\rangle:=\mathcal{T}^{+}\left|y^{+}\right\rangle=\frac{1}{\sqrt{2 \pi}} \int_{0}^{\infty} d y \frac{\sin \sqrt{s y}}{(s y)^{1 / 4}}\left|y^{+}\right\rangle, \\
& \left|s^{-}\right\rangle:=\mathcal{T}^{-}\left|y^{-}\right\rangle=\frac{1}{\sqrt{2 \pi}} \int_{0}^{\infty} d y \frac{\cos \sqrt{s y}}{(s y)^{1 / 4}}\left|y^{-}\right\rangle .
\end{aligned}
$$

for $\left|y^{ \pm}\right\rangle \in \mathcal{D}_{ \pm}^{\times}$. Here, $\mathcal{T}^{ \pm}$are the extensions of these operators to the duals. Vectors $\left|s^{ \pm}\right\rangle$could be identified as momentum-like kets, since they are Fourier-like transforms of position kets. Contrarily to $\left|y^{ \pm}\right\rangle$, kets $\left|s^{ \pm}\right\rangle$cannot be identified as they come from different transformations of two isomorphic objects.

Finally, note that similar expressions of those of vectors $\left|s^{ \pm}\right\rangle$can be obtained for all RHS of the form $\mathcal{D}_{k, r}^{ \pm} \subset L_{k, r}^{2}\left(\mathbb{R}^{+}\right)^{ \pm} \subset\left(\mathcal{D}_{k, r}^{ \pm}\right)^{\times}$.

\section{CONCLUSIONS}

The first systematic formulation of quantum mechanics was proposed by Dirac. ${ }^{34}$ In this book, bases for spaces of solutions were constructed with eigenvectors of Hermitian operators defining the observables of a quantum system. In this context, the same space could had both discrete and continuous bases. At the same time, Dirac introduced the "delta" function, which is the point of departure of the mathematical theory of distributions. However, the formulation of quantum mechanics in Hilbert spaces allows for discrete bases although not for continuous bases, which usually are given in terms of generalized functions.

Rigged Hilbert complex spaces are the rigorous mathematical structure to describe quantum mechanics beyond systems with purely discrete spectrum as is the correct framework to include discrete and continuous bases on a simple interchangeable level. Therefore, it is the correct mathematical framework for the Dirac formulation of quantum mechanics.

In addition, the need of RHS in quantum mechanics becomes apparent among other contexts: as rigorous construction of the Gamow states or vector states for unstable quantum systems (resonances) or in modern formulation of Time Asymmetric Quantum Theory (TAQT). ${ }^{35}$

RHSs used in quantum mechanics are triplets of vector spaces on the field of complex numbers. In signal theory, however, the triplet of spaces might be also on the field of real numbers.

RHS exhibit a strict relation with Lie algebras and UEAs, which is not shared by Hilbert spaces. RHS can indeed be constructed such that generators and universal enveloping elements of Lie algebras can be represented as well behaved continuous operators where problems of domains, that characterize unbounded operators, do not apply. ${ }^{9}$

A fundamental property of rigged Hilbert spaces is indeed related to the fact that they support representations of locally compact Lie groups. ${ }^{25}$ This idea could also be applied to the use of RHS as support spaces for representations of semigroups. In fact, unitary representations of semigroups in RHS have already been considered. See for instance Ref. 36 and references therein.

In particular, when the representation is irreducible, as in the cases here considered, the space of the operators defined on the RHS is isomorphic to the UEA, so that all properties of the algebra can be transferred to its representations on RHS, which acquire all the symmetries of the algebra.

In terms of possible applications, it may be relevant the decomposition of RHS into direct sums of RHS. This implies that in a physical system or in a signal we can single out - with great freedom - what we consider relevant, filtering either the noise or any other undesirable addition. The same freedom exists with the operators where we can restrict their evolution inside a subalgebra chosen among infinite others.

We have discussed two situations depending on whether wave functions and signals are supported on the whole real line $\mathbb{R}$ or on the positive semi-axis $\mathbb{R}^{+}$. For $\mathbb{R}$ we use a Hilbert space basis formed by eigenvectors of the Fourier transform. Then, fractional Fourier transforms determine a decomposition of the RHS as a direct sum of RHS. This procedure is the essence of the filtering procedure. This idea does apply also to $\mathbb{R}^{+}$. Indeed, we can introduce a pair of "Fourier-like" transformations which play the role before assigned to the Fourier transform. These Fourier-like 
transforms have their own fractional transforms that also serve for filtering purposes. In both cases, the algebraic approach allows us to extend all results from the vector spaces to the operators acting on them.

\section{ACKNOWLEDGMENTS}

This work was partially supported by the Ministerio de Economía y Competitividad of Spain (Project No. MTM2014-57129-C2-1-P with EU-FEDER support) and the Junta de Castilla y León (Project VA057U16).

${ }^{1}$ S. C. Coutinho, A Premier on Algebraic D-Modulus (Cambridge University Press, Cambridge, MA, 1995).

${ }^{2}$ G. B. Folland, Fourier Analysis and Its Applications (Pacific Grove, CA, Wadsworth, 1992).

${ }^{3}$ E. Celeghini and M. A. del Olmo, Ann. Phys. 335, 78 (2013).

${ }^{4}$ E. Celeghini and M. A. del Olmo, J. Phys.: Conf. Ser. 597, 012022 (2015).

${ }^{5}$ E. Celeghini, J. Phys.: Conf. Ser. 626, 012047 (2015).

${ }^{6}$ H. M. Ozaktas, Z. Zalevsky, and M. Alper Kutay, The Fractional Fourier Transform (Wiley, Chichester, 2001).

${ }^{7}$ W. A. Skok Narayanan and K. M. M. Prabhu, Microprocessors Microsyst. 27, 511 (2003).

${ }^{8}$ I. M. Gelfand and N. Ya. Vilenkin, Generalized Functions: Applications to Harmonic Analysis (Academic, New York, 1964).

9 J. E. Roberts, Commun. Math. Phys. 3, 98 (1966).

10 J. P. Antoine, J. Math. Phys. 10, 53 (1969).

${ }^{11}$ O. Melsheimer, J. Math. Phys. 15, 902 (1974).

12 A. Bohm, The Rigged Hilbert Space and Quantum Mechanics, Springer Lecture Notes in Physics (Springer, Berlin, 1978), Vol. 78.

${ }^{13}$ A. Bohm and M. Gadella, Dirac Kets, Gamow Vectors and Gelfand Triplets, Springer Lecture Notes in Physics (Springer, Berlin, 1989), Vol. 348.

${ }^{14}$ M. Gadella and F. Gómez, Found. Phys. 32, 815 (2002).

${ }^{15}$ This means that any Cauchy sequence in $\Phi$ on the Hilbert space norm has a limit in $\mathcal{H}$.

${ }^{16}$ J. Horvath, Topological Vector Spaces and Distributions (Addison-Wesley, Reading, MA, 1966).

${ }^{17}$ M. Gadella and F. Gómez, Int. J. Theor. Phys. 42, 2225 (2003).

${ }_{18}$ M. Gadella and F. Gómez, Acta Appl. Math. 109, 94 (2010).

${ }^{19}$ For any $\phi \in \mathcal{H}$, we can define a functional, $F_{\phi} \in \Phi^{\times}$in the following way:

$$
\left\langle\varphi \mid F_{\phi}\right\rangle:=\langle\varphi \mid \phi\rangle, \quad \forall \varphi \in \Phi .
$$

where the product in the right hand side denotes the ordinary scalar product on $\mathcal{H}$. The mapping $\phi \longmapsto F_{\phi}$ is one to one and defines a canonical injection $\mathcal{H} \longmapsto \Phi^{\times}$.

${ }^{20}$ For a thoroughly study of changes of representations involving continuous bases see Ref. 18 .

${ }^{21}$ M. Hammermesh, Group Theory (Addison-Wesley, Reading, MA, 1962).

${ }^{22}$ V. Bargmann, Ann. Math. 59, 1 (1954).

${ }^{23}$ E. Celeghini and M. Tarlini, Nuovo Cimento B 65, 172 (1981).

${ }^{24}$ Remember that we are considering antilinear mappings in our duals and not linear as customary.

${ }^{25}$ K. Maurin, General Eigenfunction Expansions and Unitary Representations of Topological Groups (Polish Scientific Publishers, Warszawa, 1968).

${ }^{26}$ A. Pietsch, Nuclear Topological Vector Spaces (Springer, Berlin, 1972).

${ }^{27}$ In fact $d \mu(\lambda)=\rho(\lambda) d \lambda$, where $\rho(\lambda)$ is the Radom-Nikodym derivative of $\mu$ with respect to the Lebesgue measure. Then, we may define a new $|\lambda\rangle$ by $\sqrt{\rho(\lambda)}|\lambda\rangle$ without loss of generality.

${ }^{28}$ For instance, $\left\langle x \mid x^{\prime}\right\rangle=\sqrt{2 \pi} \delta\left(x-x^{\prime}\right)$, which has a meaning as a kernel. ${ }^{10}$

${ }^{29}$ This converges in the strong operator sense on $L^{2}(\mathbb{R})$.

${ }^{30}$ M. Reed and B. Simon, Functional Analysis (Academic, New York, 1972).

${ }^{31}$ M. Abramovich and I. A. Stegun, Handbook of Mathematical Functions with Formulas, Graphs, and Mathematical Tables (Dover, New York, 1972).

${ }^{32}$ NIST Handbook of Mathematical Functions, edited by F. W. J. Olver, D. W. Lozier, R. F. Boisiert, and C. W. Clark (Cambridge University Press, Cambridge, MA, 2010).

${ }^{33}$ G. Lindblad and B. Nagel, Ann. Inst. Henri Poincaré 13, 27 (1970).

${ }^{34}$ P. A. M. Dirac, The Principles of Quantum Mechanics (Clarendon Press, Oxford, 1958)

35 A. Bohm, M. Gadella, and P. Kielanowski, SIGMA 7, 086 (2011).

${ }^{36}$ M. Gadella, F. Gómez-Cubillo, L. Rodríguez, and S. Wickramasekara, J. Math. Phys. 54, 072303 (2013). 